\title{
Pilot Screening to Determine Antimicrobial Synergies in a Multidrug-Resistant Bacterial Strain Library
}

\author{
Si-Hyun Kim, ${ }^{1-3}$ Chulmin Park, Hye-Sun Chun, Dong-Gun Lee, ${ }^{1,3}$ Jae-Ki Choi,,3 Hyo-Jin Lee,3 \\ Sung-Yeon Cho, ${ }^{1,3}$ Sun Hee Park, ${ }^{1,3}$ Su-Mi Choi, ${ }^{1,3}$ Jung-Hyun Choi, ${ }^{1-3}$ and Jin-Hong Yoo ${ }^{1,3}$
}

With the rise in multidrug-resistant (MDR) bacterial infections, there has been increasing interest in combinations of $\geq 2$ antimicrobial agents with synergistic effects. We established an MDR bacterial strain library to screen for in vitro antimicrobial synergy by using a broth microdilution checkerboard method and highthroughput luciferase-based bacterial cell viability assay. In total, 39 MDR bacterial strains, including 23 carbapenem-resistant gram-negative bacteria, 9 vancomycin-intermediate Staphylococcus aureus, and 7 vancomycin-resistant Enterococcus faecalis, were used to screen for potential antimicrobial synergies. Synergies were more frequently identified with combinations of imipenem plus trimethoprim-sulfamethoxazole for carbapenem-resistant Acinetobacter baumannii in the library. To verify this finding, we tested 34 A. baumannii clinical isolates resistant to both imipenem and trimethoprim-sulfamethoxazole by the checkerboard method. The imipenem plus trimethoprim-sulfamethoxazole combination showed synergy in the treatment of $21(62 \%)$ of the clinical isolates. The results indicate that pilot screening for antimicrobial synergy in the MDR bacterial strain library could be valuable in the selection of combination therapeutic regimens to treat MDR bacterial infections. Further studies are warranted to determine whether this screening system can be useful to screen for the combined effects of conventional antimicrobials and new-generation antimicrobials or nonantimicrobials.

\section{Introduction}

B ECAUSE OF WIDESPREAD use of antimicrobial drugs over several decades, antimicrobial resistance has become an increasingly serious threat to global public health. High proportions of antimicrobial resistance in bacteria, which cause common infections such as urinary tract infections, pneumonia, and bloodstream infections, have been reported in both community-acquired and hospital-acquired infections worldwide. Recently, multidrug-resistant (MDR) bacteria have emerged as major pathogens in hospitalized patients, especially in critically ill patients. These bacteria are resistant to almost all commercially available antimicrobial agents. Since therapeutic options are limited, MDR bacterial infections are more difficult to treat, resulting in extremely high mortality rates. The occurrence of antimicrobial resistance continues to outpace the development of new antimicrobial drugs, and as the prevalence of infections caused by MDR bacteria continues to increase, demand for combination antimicrobial therapies is growing rapidly.

Because synergies can be observed with the combination of $\geq 2$ antimicrobial agents, clinicians are often tempted to use combination therapies to treat MDR bacterial infections. ${ }^{8,21}$ There are several clinical indications for the use of antimicrobial combinations ${ }^{1,11,16}$; however, synergistic effects are very complicated. For example, combined antimicrobial agents may demonstrate an undesirable interaction, that is, antagonism, against a given organism. In vitro effects of antimicrobial combinations have been assessed by various methods, and each has its potential advantages and limitations. ${ }^{17}$

In this study, we established an MDR bacterial strain library for in vitro screening to detect synergies in antimicrobial combinations by using a broth microdilution

A part of this study was presented in a poster session at the 10th International Symposium on Antimicrobial Agents and Resistance (ISAAR), May 14-15, 2015, Incheon, Republic of Korea.

${ }^{1}$ Vaccine Bio Research Institute, College of Medicine, The Catholic University of Korea, Seoul, Republic of Korea.

${ }^{2}$ Division of Infectious Diseases, Department of Internal Medicine, College of Medicine, The Catholic University of Korea, Incheon St. Mary's Hospital, Incheon, Republic of Korea.

${ }^{3}$ Division of Infectious Diseases, Department of Internal Medicine, College of Medicine, The Catholic University of Korea, Seoul, Republic of Korea.

(C) Si-Hyun Kim, et al., 2016; Published by Mary Ann Liebert, Inc. This Open Access article is distributed under the terms of the Creative Commons Attribution Noncommercial License (http://creativecommons.org/licenses/by-nc/4.01/) which permits any noncommercial use, distribution, and reproduction in any medium, provided the original author(s) and the source are credited. 
checkerboard method and a high-throughput luciferasebased bacterial cell viability assay to measure fractional inhibitory concentrations.

\section{Materials and Methods}

\section{Bacterial strains}

The MDR bacterial strain library used in this study was composed of 39 MDR and 6 drug-susceptible strains. MDR bacteria included 23 carbapenem-resistant gram-negative strains, representing 4 genera (Klebsiella pneumoniae, Escherichia coli, Pseudomonas aeruginosa, and Acinetobacter baumannii), 9 vancomycin-intermediate Staphylococcus aureus (VISA), and 7 vancomycin-resistant Enterococcus faecium (VRE). Carbapenem-resistant K. pneumoniae, E. coli, P. aeruginosa, and VISA strains were obtained from Korean Centers for Disease Control and Prevention. Carbapenem-resistant $A$. baumannii and VRE strains were selected from the clinical isolates collection of the Catholic University of Korea connected hospitals (three hospitals). Four carbapenem-susceptible gram-negative strains (E. coli ATCC 25922 and ATCC 35218, P. aeruginosa ATCC 27853, and A. baumannii Aci100085 [clinical isolate]) and 2 vancomycin-susceptible gram-positive strains (S. aureus ATCC 29213 and Enterococcus faecalis ATCC 29212) were used as controls. In addition, 89 A. baumannii clinical isolates were screened for carbapenem susceptibility. Among them, carbapenem-resistant strains were used to verify synergistic effects of combination therapies.

\section{Bacterial growth conditions and susceptibility testing}

Each bacterial strain was grown on blood agar (Hanil Komed, Co., Seongnam, South Korea) or tryptic soy agar (Becton Dickinson, Sparks, MD), and the Mueller-Hinton broth (Becton Dickinson) was used for antimicrobial susceptibility testing and bacterial cell viability assay.

Susceptibility to a variety of antimicrobial agents, including amoxicillin-clavulanate (AMX), ceftazidime (CAZ), ciprofloxacin (CIP), daptomycin (DAP), gentamicin (GEN), imipenem (IMP), linezolid (LZ), trimethoprim-sulfamethoxazole (TMP/SMX), and vancomycin (VAN), was tested by a broth microdilution method, in accordance with the Clinical and Laboratory Standards Institute recommendations (2010). ${ }^{4}$ All antimicrobials were obtained from Sigma-Aldrich Korea (Seoul, South Korea).

\section{Luciferase-based bacterial cell viability assay}

BacTiter-Glo $^{\mathrm{TM}}$ microbial cell viability assay (Promega Corp., Madison, WI), a homogeneous method for determining the number of viable microbial cells in culture that is based on the quantitation of adenosine triphosphates present, was used for examining bacterial cell growth and evaluating antimicrobial activity. ${ }^{9}$ All assays were performed in opaque-walled multiwell plates, according to the manufacturer's recommendation. Bacterial cell numbers ranged from $10^{2}$ to $10^{8}$ colony-forming units (CFUs) among strains used for the assay. The luminescent signal, interpreted as the relative luminescence unit (RLU), was measured using a SpectraMax L Microplate Reader (Molecular Devices, Sunnyvale, CA). Reported signals represent the mean of three replicates. The signal-to-noise ratio ( $\mathrm{S}: \mathrm{N}$ ratio) was calculated as follows: $\mathrm{S}: \mathrm{N}$ ratio $=($ mean of signal - mean of background)/standard deviation of background. The data were used for constructing growth curves, for susceptibility testing, and rapid screening of combined antimicrobial effects in culture by the checkerboard method, and for determining growth of $P$. aeruginosa and $A$. baumannii strains in the presence of TMP/SMX.

\section{Antimicrobial resistance genes in MDR bacterial strains}

All MDR gram-negative bacteria were screened for the presence of $b l a_{\mathrm{NDM}-1}, b l a_{\mathrm{GES}}, b l a_{\mathrm{IMP}}, b l a_{\mathrm{VIM}}, b l a_{\mathrm{AmpC}}$, and $b l a_{\mathrm{KPC}}$ by polymerase chain reaction (PCR) and for bla $a_{\mathrm{OXA}}$ by multiplex PCR by using the previously described primers. $3,10,12,13,20$ The primer sequences of the genes used in this study are listed in Table 1.

All MDR gram-positive bacterial strains were screened by multiplex PCR for the presence of mecA and vancomycin resistance genes, as previously described. ${ }^{6,15}$

\section{Checkerboard synergy test}

A screening test for potential synergies was conducted using a broth microdilution checkerboard method. All assays were performed in triplicate with 96-well U-bottom microtiter trays in an eight-by-eight-well configuration. ${ }^{2,7}$ Each antimicrobial was twofold diluted with the concentration ranging from $0.063 \times \mathrm{MIC}$ to $4 \times \mathrm{MIC}$. The gradient concentration of each antimicrobial was mixed in each well. Each well was inoculated with $5 \times 10^{5} \mathrm{CFUs}$ of a bacterial strain in the cation-adjusted Mueller-Hinton broth. Positive growth controls that did not contain any antibiotics and negative growth controls were included in each assay. The combination of IMP plus GEN, CIP, or TMP/SMX was used for the pilot screening of synergistic activity against carbapenem-resistant gram-negative strains. For VISA and VRE, the combination of VAN plus GEN or CIP was tested. Microtiter trays were incubated for $18-24 \mathrm{hr}$ at $35^{\circ} \mathrm{C}$ in ambient air. After incubation, growth was determined using microbial cell viability assay.

The total fractional inhibitory concentration ( $\Sigma$ FIC, FIC index), used to interpret the checkerboard synergy test, was calculated according to the following formula: $\Sigma \mathrm{FIC}=\mathrm{FIC}$ of drug A+FIC of drug B. In this formula, FIC of drug A and FIC of drug B were calculated as follows: FIC of drug A $($ or $\mathrm{B})=$ minimum inhibitory concentration $(\mathrm{MIC})$ of $\operatorname{drug} \mathrm{A}$ (or B) in combination/MIC of drug A (or B) alone. The results of the FIC index were defined as follows: $\Sigma$ FIC $\leq 0.5$, synergy; $0.5<\Sigma F I C \leq 1$, additivity; $1<\Sigma F I C \leq 4$, indifference; and $\Sigma \mathrm{FIC}>4$, antagonism. ${ }^{2,14}$

\section{Statistical analysis}

Fisher's exact test was used to compare categorical variables. Two-tailed $p$-values of $<0.05$ were considered statistically significant. We used SPSS software version 18.0 (SPSS Korea, Seoul, Korea) for the statistical analysis.

\section{Results}

\section{MICs and growth curves of bacterial strains in the MDR bacterial strain library}

All bacterial strains in the MDR bacterial strain library were tested for antimicrobial susceptibility to single 
Table 1. Polymerase Chain Reaction Primers for Antimicrobial Resistance Genes in Multidrug-Resistant Gram-Negative Bacteria

\begin{tabular}{|c|c|c|c|}
\hline Gene & Primer & Sequence $\left(5^{\prime} \rightarrow 3^{\prime}\right)$ & Reference \\
\hline \multirow{2}{*}{$b l a_{\mathrm{NDM}-1}$} & NDM-F & TTGGCCTTGCTGTCCTTG & 13 \\
\hline & NDM-R & ACACCAGTGACAATATCACCG & \\
\hline \multirow{2}{*}{$b l a_{\mathrm{GES}}$} & GES-F & CTATTACTGGCAGGGATCG & 13 \\
\hline & GES-R & CCTCTCAATGGTGTGGGT & \\
\hline \multirow{2}{*}{$b l a_{\mathrm{IMP}}$} & IMP-F & GAGTGGCTTAATTCTCRATC & 10 \\
\hline & IMP-R & AACTAYCCAATAYRTAAC & \\
\hline \multirow[t]{2}{*}{$b l a_{\mathrm{VIM}}$} & VIM-F & GTTTGGTCGCATATCGCAAC & 10 \\
\hline & VIM-R & AATGCGCAGCACCAGGATAG & \\
\hline \multirow[t]{2}{*}{$b l a_{\mathrm{AmpC}}$} & AmpC-F & ACTTACTTCAACTCGCGACG & 4 \\
\hline & AmpC-R & TAAACACCACATATGTTCCG & \\
\hline \multirow[t]{2}{*}{$b l a_{\mathrm{KPC}}$} & KPC-F & TCGCTAAACTCGAACAGG & 12 \\
\hline & KPC-R & TTACTGCCCGTTGACGCCCAATCC & \\
\hline \multirow{2}{*}{$b l a_{\mathrm{OXA}-23-\mathrm{like}}$} & OXA-23-like-F & GATCGGATTGGAGAACCAGA & 20 \\
\hline & OXA-23-like-R & ATTTCTGACCGCATTTCCAT & \\
\hline \multirow{2}{*}{$b l a_{\mathrm{OXA}-24-\mathrm{like}}$} & OXA-24-like-F & GGTTAGTTGGCCCCCTTAAA & 20 \\
\hline & OXA-24-like-R & AGTTGAGCGAAAAGGGGATT & \\
\hline \multirow[t]{2}{*}{$b l a_{\text {OXA-51-like }}$} & OXA-51-like-F & TAATGCTTTGATCGGCCTTG & 20 \\
\hline & OXA-51-like-R & TGGATTGCACTTCATCTTGG & \\
\hline \multirow{2}{*}{$b_{\text {OXA-58-like }}$} & OXA-58-like-F & AAGTATTGGGGCTTGTGCTG & 20 \\
\hline & OXA-58-like-R & CCCCTCTGCGCTCTACATAC & \\
\hline
\end{tabular}

antimicrobial agents by growth curve analysis. MIC ranges of 23 carbapenem-resistant gram-negative, 9 VISA, and 7 VRE strains are presented in Table 2.

The bacterial cell viability assay showed that the S:N ratio correlated with the bacterial cell number (Fig. 1). The percentage of RLU (\%RLU) relative to the positive growth controls correlated with results of the conventional MIC assay. Growth curves were also used to determine $>2-\log$ kill dose of a drug alone or in combination.

\section{Antimicrobial resistance genes in MDR bacterial strains}

The characteristics of antimicrobial resistance in MDR bacterial strains are listed in Tables 3 and 4. Three of seven (43\%) carbapenem-resistant K. pneumoniae strains carried $b l a_{\mathrm{KPC}}$. Of the eight carbapenem-resistant $P$. aeruginosa, $b l a_{\mathrm{IMP}}$ was detected in six strains (75\%). Two distinct $b l a_{\mathrm{OXA}^{-}}$ like genes, $b l a_{\text {OXA-23-like }}$ and $b l a_{\text {OXA-51-like, were identified }}$ in five (71\%) and two (29\%) of seven carbapenem-resistant $A$. baumannii strains, respectively. All carbapenem-resistant $A$. baumannii strains contained $b l a_{\mathrm{AmpC}}$. All VISA strains carried $m e c A$, but no vancomycin resistance genes. All VRE strains carried vanA.

\section{Pilot screening of combination effects in the MDR bacterial strain library}

Antimicrobial agents were screened for combination effects against all 39 MDR bacterial strains. Based on the MIC and known mechanism of action of each antimicrobial agent, the combinations of IMP plus GEN, CIP, or TMP/ SMX were the preferred regimens in the pilot screening for synergistic activity against carbapenem-resistant gramnegative strains. For VISA and VRE strains, combinations of VAN plus GEN or CIP were tested for synergistic effects.

The characteristics and $\Sigma F I C$ values of 23 MDR gramnegative strains are shown in Table 3 . Regardless of the combination regimen, additivity was the most common effect observed in all 23 strains $(37 / 69,54 \%)$. None of the antimicrobial combinations showed antagonistic activity against any of the strains. Overall, the combination of IMP plus TMP/SMX demonstrated synergy most often against the carbapenem-resistant bacterial strains $(7 / 23,30 \%)$.

Table 2. MIC Ranges of Carbapenem-Resistant Gram-Negative Strains, Vancomycin-Intermediate STAPHYLOCOCCUS AUREUS, AND VANCOMYCIN-RESISTANT ENTEROCOCCUS FAECIUM

\begin{tabular}{|c|c|c|c|c|c|c|c|c|c|}
\hline \multirow[b]{2}{*}{ Organism (no. tested) } & \multicolumn{9}{|c|}{ MIC range $(\mu \mathrm{g} / \mathrm{mL})$ for } \\
\hline & $I M P$ & $A M X$ & $C A Z$ & $C I P$ & $G E N$ & $T M P / S M X$ & $V A N$ & $D A P$ & $L Z$ \\
\hline $\begin{array}{l}\text { Carbapenem-resistant } \\
\text { gram-negative strains (23) }\end{array}$ & $4->128$ & $>128 / 64$ & $64->128$ & $8->128$ & $8->128$ & $4 / 76-\geq 128 / 2432$ & - & - & - \\
\hline VISA (9) & - & - & - & $16-32$ & $64->512$ & - & $2-4$ & $2-8$ & $0.5-2$ \\
\hline VRE (7) & - & - & - & $128-256$ & $>512$ & - & $16->128$ & $4-32$ & $1-2$ \\
\hline
\end{tabular}

VISA, vancomycin-intermediate Staphylococcus aureus; VRE, vancomycin-resistant Enterococcus faecium; AMX, amoxicillinclavulanate; CAZ, ceftazidime; CIP, ciprofloxacin; DAP, daptomycin; GEN, gentamicin; IMP, imipenem; LZ, linezolid; TMP/SMX, trimethoprim-sulfamethoxazole; VAN, vancomycin; MIC, minimum inhibitory concentration. 

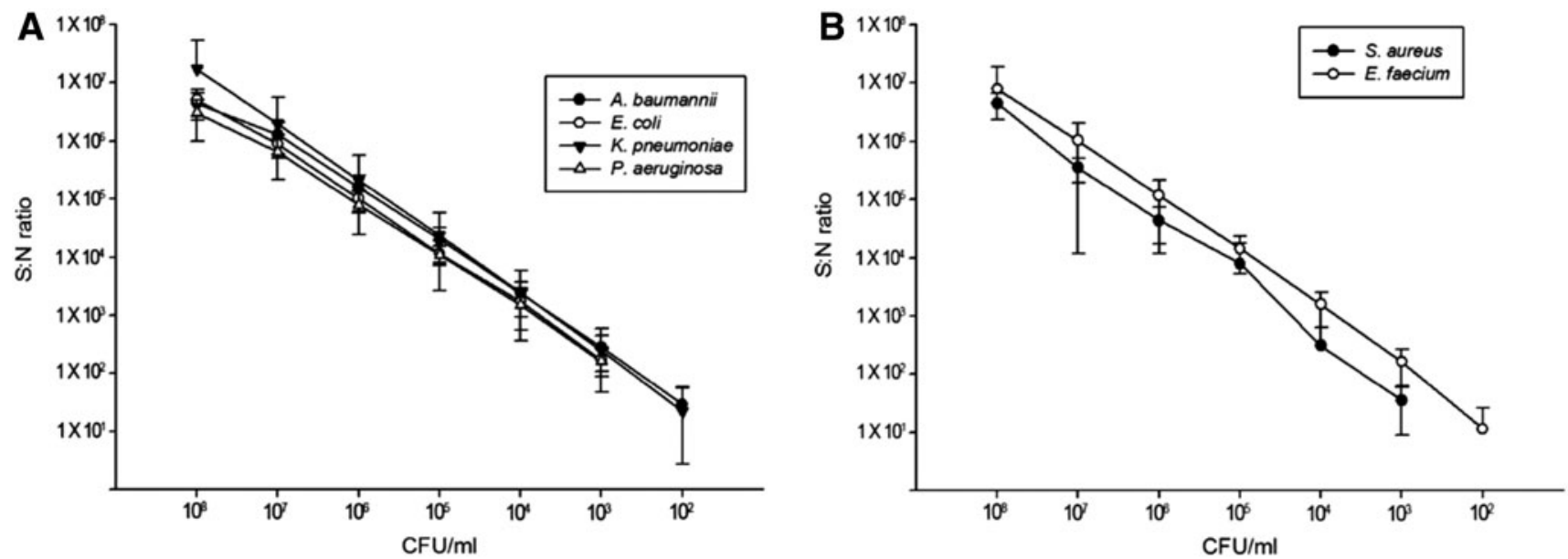

FIG. 1. Determination of bacterial cell numbers by a luciferase-based microbial cell viability assay. Multidrug-resistant gram-negative (A) and gram-positive bacterial strains (B). Results show a linear correlation between the luminescent signal and the number of bacterial cells, which ranged from $10^{2}$ to $10^{8}$ colony-forming units (CFUs).

Synergistic or additive effects of IMP combined with TMP/ SMX were observed in all seven carbapenem-resistant $K$. pneumoniae strains. For the eight carbapenem-resistant $P$. aeruginosa strains, the combination of IMP plus CIP showed the lowest FIC indices for 10\%, 50\%, and 90\% of the isolates $(0.375,0.625$, and 1.001 , respectively), compared with the FIC indices of other antimicrobial combinations (0.501, 1.000 , and 1.500 for IMP plus GEN; 0.563, 1.500, and 2.000 for IMP plus TMP/SMX). Combinations of IMP plus CIP and IMP plus TMP/SMX both exhibited synergies against one $E$. coli bla $a_{\mathrm{NDM}-1}$ strain. For the seven carbapenem-resistant $A$. baumannii strains, the synergies observed differed significantly between combination regimens ( 2 of 7 [29\%] for IMP plus GEN, 0 of 7 [0\%] for IMP plus CIP, and 5 of 7 [71\%] for IMP plus TMP/SMX; $p=0.025$ ).

Table 4 shows the characteristics and $\Sigma F I C$ values of 16 MDR gram-positive strains. Both the combinations of VAN plus GEN and VAN plus CIP showed synergistic effects against one of nine VISA strains (11\%). For the seven VRE strains, synergy was detected more often in the combination of VAN plus CIP (100\%) than in the combination of VAN plus GEN $(29 \% ; p=0.021)$.

Table 3. Total Fractional Inhibitory Concentrations of Combinations of Antimicrobial Agents Against Carbapenem-Resistant KLEBSiella PNEUmoniae, Escherichia Coli, PSEUdomonas aERUGinosa, and Acinetobacter baUmannit Strains Obtained by the Checkerboard Method

\begin{tabular}{|c|c|c|c|c|c|}
\hline Strain & Species & Characteristics & IMP plus GEN & IMP plus CIP & IMP plus TMP/SMX \\
\hline cmkc0001 & K. pneumoniae & NDM-1 & $1.5(\mathrm{I})$ & $0.75(\mathrm{~A})$ & $0.25(\mathrm{~S})$ \\
\hline cmkc0002 & K. pneumoniae & KPC-2 & $0.625(\mathrm{~A})$ & $2(\mathrm{I})$ & $1(\mathrm{~A})$ \\
\hline cmkc0003 & K. pneumoniae & KPC-2 & $0.5(\mathrm{~S})$ & $2(\mathrm{I})$ & $1(\mathrm{~A})$ \\
\hline cmkc0004 & K. pneumoniae & KPC-2 & $0.5(\mathrm{~S})$ & $0.263(\mathrm{~S})$ & $1(\mathrm{~A})$ \\
\hline cmkc0005 & K. pneumoniae & GES-5 & $1(\mathrm{~A})$ & $0.75(\mathrm{~A})$ & $1(\mathrm{~A})$ \\
\hline cmkc0006 & K. pneumoniae & IMP-1 & $1(\mathrm{~A})$ & $0.625(\mathrm{~A})$ & $1(\mathrm{~A})$ \\
\hline cmnc0014 & K. pneumoniae & NDM-1 & $1(\mathrm{~A})$ & $0.313(\mathrm{~S})$ & $0.75(\mathrm{~A})$ \\
\hline cmnc0007 & E. coli & NDM-1 & 1 (A) & $0.375(\mathrm{~S})$ & $0.375(\mathrm{~S})$ \\
\hline cmkc0008 & P. aeruginosa & IMP-1 & $1(\mathrm{~A})$ & $0.625(\mathrm{~A})$ & 2 (I) \\
\hline cmkc0009 & $P$. aeruginosa & IMP-6 & $0.501(\mathrm{~A})$ & $0.625(\mathrm{~A})$ & 2 (I) \\
\hline cmkc0010 & P. aeruginosa & VIM-2 & $1(\mathrm{~A})$ & $0.501(\mathrm{~A})$ & $1.5(\mathrm{I})$ \\
\hline cmkc0011 & P. aeruginosa & VIM-2 & $1(\mathrm{~A})$ & 1.001 (I) & $0.625(\mathrm{~A})$ \\
\hline cmnp0015 & P. aeruginosa & IMP-1 & 1.001 (I) & $0.625(\mathrm{~A})$ & $2(\mathrm{I})$ \\
\hline cmnp0016 & P. aeruginosa & IMP-1 & 1 (A) & $0.375(\mathrm{~S})$ & $1(\mathrm{~A})$ \\
\hline cmnp0017 & P. aeruginosa & IMP-1 & $1.001(\mathrm{I})$ & $0.625(\mathrm{~A})$ & $2(\mathrm{I})$ \\
\hline cmnp0018 & $P$. aeruginosa & IMP-1 & 1.5 (I) & $0.5(\mathrm{~S})$ & $0.563(\mathrm{~A})$ \\
\hline cmdr0054 & A. baumannii & OXA-23-like, AmpC & $0.375(\mathrm{~S})$ & $0.75(\mathrm{~A})$ & $0.375(\mathrm{~S})$ \\
\hline cmdr0055 & A. baumannii & OXA-23-like, AmpC & $1.5(\mathrm{I})$ & $0.625(\mathrm{~A})$ & $0.5(\mathrm{~S})$ \\
\hline cmdr0056 & A. baumannii & OXA-23-like, AmpC & $1.5(\mathrm{I})$ & 1 (A) & $0.375(\mathrm{~S})$ \\
\hline cmdr0057 & A. baumannii & OXA-23-like, AmpC & $0.625(\mathrm{~A})$ & $1(\mathrm{~A})$ & $0.375(\mathrm{~S})$ \\
\hline cmdr0058 & A. baumannii & OXA-23-like, AmpC & $0.625(\mathrm{~A})$ & $1(\mathrm{~A})$ & $0.5(\mathrm{~S})$ \\
\hline cmdr0059 & A. baumannii & OXA-51-like, AmpC & $1(\mathrm{~A})$ & $1.5(\mathrm{I})$ & $1(\mathrm{~A})$ \\
\hline cmdr0060 & A. baumannii & OXA-51-like, AmpC & $0.375(\mathrm{~S})$ & 1.001 (I) & $0.75(\mathrm{~A})$ \\
\hline
\end{tabular}

A, additivity; I, indifference; S, synergy. 
TABle 4. Total Fractional Inhibitory

CONCENTRATIONS OF COMBINATIONS OF ANTIMICROBIAL AgEnts Against VANCOMYCIN-INTERMEdiate

S. AUREUS AND VANCOMYCIN-RESISTANT E. FAECIUM

Strains Obtained by the Checkerboard Method

\begin{tabular}{|c|c|c|c|c|}
\hline Strain & Species & Characteristics & $\begin{array}{l}\text { VAN } \\
\text { plus } \\
G E N\end{array}$ & $\begin{array}{l}\text { VAN } \\
\text { plus } \\
\text { CIP }\end{array}$ \\
\hline cmnc0020 & S. aureus & VISA & $2(\mathrm{I})$ & $1(\mathrm{~A})$ \\
\hline cmnc0021 & S. aureus & VISA & 1 (A) & $1(\mathrm{~A})$ \\
\hline cmnc0022 & S. aureus & VISA & 0.563 (A) & $0.625(\mathrm{~A})$ \\
\hline cmnc0023 & S. aureus & VISA & $0.75(\mathrm{~A})$ & 0.75 (A) \\
\hline cmnc0024 & S. aureus & VISA & $0.25(\mathrm{~S})$ & $0.625(\mathrm{~A})$ \\
\hline cmnc0045 & S. aureus & VISA & 0.75 (A) & $2(\mathrm{I})$ \\
\hline cmnc0046 & S. aureus & VISA & $1(\mathrm{~A})$ & $1.5(\mathrm{I})$ \\
\hline cmnc0047 & S. aureus & VISA & 2 (I) & 0.75 (A) \\
\hline cmnc0048 & S. aureus & VISA & 2 (I) & $0.313(\mathrm{~S})$ \\
\hline cmdr0065 & E. faecium & $\operatorname{van} \mathrm{A}$ & 2 (I) & 0.75 (A) \\
\hline BM4147 & E. faecium & $\operatorname{van} \mathrm{A}$ & $0.501(\mathrm{~A})$ & $0.156(\mathrm{~S})$ \\
\hline cmdr0067 & E. faecium & $\operatorname{van} \mathrm{A}$ & $0.375(\mathrm{~S})$ & $0.501(\mathrm{~A})$ \\
\hline cmdr0069 & E. faecium & $\operatorname{van} \mathrm{A}$ & $2(\mathrm{I})$ & $0.531(\mathrm{~A})$ \\
\hline cmdr0070 & E. faecium & $\operatorname{van} \mathrm{A}$ & $2(\mathrm{I})$ & $0.75(\mathrm{~A})$ \\
\hline cmdr0071 & E. faecium & $\operatorname{van} \mathrm{A}$ & $3(\mathrm{I})$ & $0.625(\mathrm{~A})$ \\
\hline cmdr0072 & E. faecium & $\operatorname{van} \mathrm{A}$ & 2 (I) & $1(\mathrm{~A})$ \\
\hline
\end{tabular}

Synergistic effects against A. baumannii clinical isolates

Based on the pilot screening results, in which the combination of IMP plus TMP/SMX exhibited synergy most frequently $(71 \%)$ in carbapenem-resistant $A$. baumannii strains, the $\Sigma$ FIC of this combination regimen was confirmed by testing against $A$. baumannii clinical isolates. In addition, the $>2-\log$ kill dose was confirmed by growth curves of $A$. baumannii determined by the bacterial cell viability assay.

The MICs of IMP and TMP/SMX were established by a broth microdilution test. Of 89 total A. baumannii clinical isolates, 52\% (46/89), 55\% (49/89), and 38\% (34/89) were resistant to IMP, TMP/SMX, and both IMP and TMP/SMX, respectively. Twenty-eight strains $(31 \%)$ were susceptible to both. Among these isolates, the 34 clinical isolates resistant to both IMP and TMP/SMX were selected for the checkerboard test. The cumulative incidence of 34 A. baumannii clinical isolates in the FIC index of IMP plus TMP/SMX is shown in Table 5. The FIC indices of IMP plus TMP/SMX ranged from 0.313 to 2.000 , and the FIC indices for $50 \%$ and $90 \%$ of the isolates were 0.500 and 0.750 , respectively. The combination of IMP plus TMP/SMX exhibited synergy or additivity in $31(91 \%)$ isolates. Indifference and antagonism were observed in $3(9 \%)$ and $0(0 \%)$ isolates, respectively.

\section{Discussion}

In this study, we established an MDR bacterial strain library that included 39 MDR bacterial strains and 6 susceptible strains. We tested these strains for combination effects of IMP or VAN with GEN, CIP, or TMP/SMX by using the broth microdilution checkerboard method and a luciferase-based bacterial cell viability assay. The pilot screening tests showed synergy or additivity between IMP and GEN, CIP, or TMP/SMX in tests against most carbapenemresistant gram-negative bacteria and between VAN and CIP against all VRE strains. The combination of IMP plus CIP was highly effective against carbapenem-resistant $P$. aeruginosa. In particular, synergistic activity of IMP plus TMP/ SMX against carbapenem-resistant A. baumannii was remarkable. We performed synergy testing against 34 A. baumannii clinical isolates resistant to both IMP and TMP/ SMX, and synergistic or additive effects were seen against most of these isolates $(91 \%)$.

Because of the growing incidence of resistance against $\beta$ lactam antibiotics, carbapenem is frequently used as the primary treatment for gram-negative bacterial infections. Consequently, carbapenem resistance in gram-negative bacteria has emerged as a major threat to hospitalized patients and has contributed to higher rates of treatment failure and mortality due to inappropriate antimicrobial therapies. ${ }^{5,18,19}$ Vancomycin resistance in gram-positive bacteria has also received attention because of the extremely limited treatment options for these strains. The emergence and spread of these resistant strains has made monotherapies of carbapenem or vancomycin inappropriate.

The MDR bacterial strain library that was established in this study included carbapenem-resistant gram-negative bacteria (4 genera, 23 strains) and vancomycin-resistant gram-positive bacteria ( 2 genera, 16 strains). This library was used not only to evaluate the combined effects of $\geq 2$ antimicrobials in bacteria at the species level but also to assess these effects in individual isolates. We observed that synergies of certain antimicrobial combinations were not "all or none" phenomena in a single species.

In vitro evaluations of synergy between antimicrobials can inform the selection of combination antimicrobial therapies for MDR bacterial infections. Various in vitro methods have been used to detect synergies, and each method has strengths and weaknesses. Among these methods, the checkerboard and time-kill methods are the most widely used. Despite the advantage of allowing an assessment of bactericidal activity, the time-kill assay is a timeconsuming and laborious process. Thus, the time-kill assay cannot be used routinely to test many strains, as is required in a clinical setting. In comparison, the microbial cell viability assay is more convenient, less labor intensive than the

Table 5. Cumulative Incidence of 34 A. Baumannit Clinical Isolates in the Total Fractional Inhibitory Concentration of Imipenem Plus Trimethoprim-Sulfamethoxazole

\begin{tabular}{|c|c|c|c|c|c|c|c|c|c|}
\hline \multirow[b]{2}{*}{ FIC index } & \multirow{2}{*}{$\frac{\text { Interpretation }}{\text { (Value })}$} & \multicolumn{4}{|c|}{ Synergy } & \multicolumn{2}{|c|}{ Additivity } & \multicolumn{2}{|c|}{ Indifference } \\
\hline & & 0.313 & 0.325 & 0.375 & 0.5 & 0.625 & 0.75 & 1.5 & 2 \\
\hline \multicolumn{2}{|c|}{ No. of isolates (\%) } & $1(3)$ & $3(9)$ & $9(26)$ & $21(62)$ & $26(76)$ & $31(91)$ & $33(97)$ & $34(100)$ \\
\hline
\end{tabular}

FIC, fractional inhibitory concentration. 
time-kill assay, and can be used in conjunction with the checkerboard method. In fact, increases or decreases in microbial activity determined by the checkerboard test can be confirmed by the microbial cell viability assay.

Representatives of various antimicrobial classes, which differ in their targets and mechanisms of action, were used to screen combinations of carbapenem or vancomycin with other antimicrobials. Considering the MICs and different mechanisms of action, GEN, CIP, and TMP/SMX were selected to screen for combination effects with IMP or VAN. Specifically, our pilot screening suggested that IMP and TMP/SMX, which inhibit both de novo folate biosynthesis and metabolism, might show greater synergism than other antimicrobial combinations against carbapenemresistant $A$. baumannii strains. When we tested for this combination effect in $A$. baumannii clinical isolates that were resistant to both IMP and TMP/SMX, synergy or additivity was observed in $91 \%$ of the isolates.

Our study has some limitations. The number of representatives of some bacterial species, such as E. coli, was too small. Additional combinations, including new and diverse antimicrobials, could be evaluated. However, the aim of this study was to establish a platform for in vitro evaluation of combined therapies against an MDR bacterial strain library and to demonstrate convenient methods, including the checkerboard and microbial cell viability assay, for use in these evaluations. This platform may be useful for in vitro selection of combinations of antimicrobials and/or nonantimicrobials in upcoming studies.

In conclusion, the combination of IMP plus TMP/SMX showed synergistic activity against carbapenem-resistant $A$. baumannii strains in an MDR bacterial strain library, using the checkerboard method and microbial cell viability assay. The pilot screening results were verified by testing against A. baumannii clinical isolates resistant to both IMP and TMP/SMX. These findings suggest that our pilot screening against this MDR bacterial strain library can indicate promising combination therapies for use against clinical strains.

\section{Acknowledgment}

This research was supported by a Korea Health Technology R\&D Project grant (grant number: HI12C0756) through the Korea Health Industry Development Institute (KHIDI), funded by the Ministry of Health \& Welfare, Republic of Korea.

\section{Disclosure Statement}

No competing financial interests exist.

\section{References}

1. Baddour, L.M., W.R. Wilson, A.S. Bayer, V.G. Fowler, A.F. Jr., Bolger, M.E. Levison, P. Ferrieri, M.A. Gerber, L.Y. Tani, M.H. Gewitz, et al. 2005. Infective endocarditis: diagnosis, antimicrobial therapy, and management of complications: a statement for healthcare professionals from the Committee on Rheumatic Fever, Endocarditis, and Kawasaki Disease, Council on Cardiovascular Disease in the Young, and the Councils on Clinical Cardiology, Stroke, and Cardiovascular Surgery and An- esthesia, American Heart Association: endorsed by the Infectious Diseases Society of America. Circulation. 111: e394-e434.

2. Bonapace, C.R., R.L. White, L.V. Friedrich, and J.A. Bosso. 2000. Evaluation of antibiotic synergy against Acinetobacter baumannii: a comparison with Etest, time-kill, and checkerboard methods. Diagn. Microbiol. Infect. Dis. 38:43-50.

3. Bou, G., and J. Martinez-Beltran. 2000. Cloning, nucleotide sequencing, and analysis of the gene encoding an AmpC beta-lactamase in Acinetobacter baumannii. Antimicrob Agents Chemother. 44:428-432.

4. Clinical and Laboratory Standards Institute. 2010. Performance Standards for Antimicrobial Susceptibility Testing: sixteenth informational supplement M100-S16. CLSI, Wayne, PA.

5. Dantas, R.C., M.L. Ferreira, P.P. Gontijo-Filho, and R.M. Ribas. 2014. Pseudomonas aeruginosa bacteraemia: independent risk factors for mortality and impact of resistance on outcome. J. Med. Microbiol. 63:1679-1687.

6. Dutka-Malen, S., S. Evers, and P. Courvalin. 1995. Detection of glycopeptide resistance genotypes and identification to the species level of clinically relevant enterococci by PCR. J. Clin. Microbiol. 33:24-27.

7. Elemam, A., J. Rahimian, and M. Doymaz. 2010. In vitro evaluation of antibiotic synergy for polymyxin B-resistant carbapenemase-producing Klebsiella pneumoniae. J. Clin. Microbiol. 48:3558-3562.

8. Fischbach, M.A. 2011. Combination therapies for combating antimicrobial resistance. Curr. Opin. Microbiol. 14: 519-523.

9. Hunter, D.M., and D.V. Lim. 2010. Rapid detection and identification of bacterial pathogens by using an ATP bioluminescence immunoassay. J. Food Prot. 73:739-746.

10. Mendes, R.E., K.A. Kiyota, J. Monteiro, M. Castanheira, S.S. Andrade, A.C. Gales, A.C. Pignatari, and S. Tufik. 2007. Rapid detection and identification of metallo-beta-lactamase-encoding genes by multiplex realtime PCR assay and melt curve analysis. J. Clin. Microbiol. 45:544-547.

11. Micek, S.T., E.C. Welch, J. Khan, M. Pervez, J.A. Doherty, R.M. Reichley, and M.H. Kollef. 2010. Empiric combination antibiotic therapy is associated with improved outcome against sepsis due to Gram-negative bacteria: a retrospective analysis. Antimicrob Agents Chemother. 54: 1742-1748

12. Monteiro, J., A.F. Santos, M.D. Asensi, G. Peirano, and A.C. Gales. 2009. First report of KPC-2-producing Klebsiella pneumoniae strains in Brazil. Antimicrob Agents Chemother. 53:333-334.

13. Monteiro, J., R.H. Widen, A.C. Pignatari, C. Kubasek, and S. Silbert. 2012. Rapid detection of carbapenemase genes by multiplex real-time PCR. J. Antimicrob Chemother. 67:906-909.

14. Moody, J.A. 2007. Synergism Testing: Broth Microdilution Checkerboard and Broth Macrodilution Methods. ASM Press, Washington, DC, pp. 5.12.1-5.12.23.

15. Oliveira, D.C., and H. de Lencastre. 2002. Multiplex PCR strategy for rapid identification of structural types and variants of the mec element in methicillin-resistant Staphylococcus aureus. Antimicrob Agents Chemother. 46: 2155-2161.

16. Paul, M., V. Shani, E. Muchtar, G. Kariv, E. Robenshtok, and L. Leibovici. 2010. Systematic review and 
meta-analysis of the efficacy of appropriate empiric antibiotic therapy for sepsis. Antimicrob Agents Chemother. 54:4851-4863.

17. Pillai, S.K., G.M. Eliopoulos. 2005. Antimicrobial Combinations. Antibiotics in Laboratory Medicine. Fifth ed. Lippincott, Williams \& Wilkins, Baltimore.

18. Sostarich, A.M., D. Zolldann, H. Haefner, R. Luetticken, R. Schulze-Roebecke, and S.W. Lemmen. 2008. Impact of multiresistance of gram-negative bacteria in bloodstream infection on mortality rates and length of stay. Infection. 36:31-35.

19. Vardakas, K.Z., P.I. Rafailidis, A.A. Konstantelias, and M.E. Falagas. 2013. Predictors of mortality in patients with infections due to multi-drug resistant Gram negative bacteria: the study, the patient, the bug or the drug? J. infect. 66:401-414.

20. Woodford, N., M.J. Ellington, J.M. Coelho, J.F. Turton, M.E. Ward, S. Brown, S.G. Amyes, and D.M. Livermore 2006. Multiplex PCR for genes encoding preva- lent OXA carbapenemases in Acinetobacter spp. Int. J. Antimicrob Agents. 27:351-353.

21. Worthington, R.J., and C. Melander. 2013. Combination approaches to combat multidrug-resistant bacteria. Trends Biotechnol. 31:177-184.

Address correspondence to: Dong-Gun Lee, MD, PhD Division of Infectious Diseases Department of Internal Medicine College of Medicine

The Catholic University of Korea 222, Banpo-daero, Seocho-gu Seoul 137-701 Republic of Korea

E-mail: symonlee@catholic.ac.kr 